Ecography

February 2008, Volume 31, Issue 1 : Pages 16-20

http://dx.doi.org/10.1111/j.2007.0906-7590.05231.x

(c) 2008 Blackwell Publishing, Inc.
Archimer, archive institutionnelle de l'Ifremer http://www.ifremer.fr/docelec/

The definitive version is available at www.blackwell-synergy.com

\title{
Connectance and parasite diet breadth in flea-mammal webs
}

\author{
David Mouillott, ${ }^{1,}$, Boris R. Krasnov ${ }^{2}$, Georgy I. Shenbrot ${ }^{2}$ and Robert Poulin ${ }^{3}$ \\ ${ }^{1}$ UMR CNRS-IFREMER-UM2 5119 Ecosystèmes Lagunaires, University of Montpellier 2, CC 093, FR-34095 \\ Montpellier Cedex 5, France \\ ${ }^{2}$ Ramon Science Center and Mitrani Dept of Desert Ecology, Jacob Blaustein Institut for Desert Research, Ben- \\ Gurion University of the Negev, P.O.Box 194, IL-80600 Mizpe Ramon, Israel \\ ${ }^{3}$ Dept of Zoology, University of Otago, P.O. Box 56, Dunedin, New Zealand \\ *: Corresponding author : D. Mouillot, email address : mouillot@univ-montp2.fr
}

\begin{abstract}
:
The number of links in webs of species interactions, which lies at the heart of the biodiversity-stability debate, has given rise to controversy during the last $20 \mathrm{yr}$. Studies exploring these web properties have mainly focused on symmetric webs where each species can potentially feed on any other species; asymmetric webs such as host-parasite webs, where one set of species feed on another set of species, have been overlooked. However, food webs are incomplete without parasites and the study of parasite-host sub-web properties deserves attention. Here, using a large database involving 33 regional interaction webs between mammals and their flea parasites, we found a negative relationship between species richness and host-parasite connectance. We suggest that some phylogenetic constraints on flea diet may explain our observed patterns because we found that parasite diet breadth, measured as host taxonomic diversity, was invariant along our host richness gradient. We found that the slope of the logarithmic relationship between the number of realized links and species richness is lower than slope values reported for food webs. We suggest that connectance may not respond to increasing species richness as rapidly in host-parasite webs as in predator-prey food webs due to stronger coevolutionary requirements.
\end{abstract}




\section{Introduction}

Despite the extraordinary complexity of natural systems, ecologists still believe that some relatively simple laws may govern the structure of species interaction webs. Indeed the level of connectance in species interaction webs $(C)$, which is simply the ratio between realized or observed connections $(L)$ and the total number of potential connections $(T)$ among species forming the web, has been a major issue in ecology. After many other contradictory investigations ( Cohen and Briand 1984, Warren 1989, Martinez 1991, Winemiller 1989) the new paradigm of food web theory is that connectance decreases with species richness (Schmid-Araya et al. 2002, Beckerman et al. 2006).

However, studies exploring this phenomenon have mainly focused on webs of undirected links where each species can potentially feed on every other species (symmetric webs) through trophic interactions. Other types of webs present in nature have been largely overlooked, except for mutualistic networks such as plant-pollinator webs (e.g. Jordano 1987). Parasitism, which is the most common consumer-resource interaction, generates asymmetric webs where one set of species feed on another set of species. Insect parasitoids are the only type of parasite commonly used to investigate the structure of such asymmetric webs (Memmott et al. 2000, Lewis et al. 2002) because parasitoids are large relative to host size and are easy to sample. By contrast, host-parasite webs, which are also asymmetric webs, have been mostly neglected although parasites constitute a fundamental component of food webs (Marcogliese and Cone 1997; Thompson et al. 2005; Lafferty et al. 2006). Most species on earth harbour parasites and more than half the known animals can be parasitic at some points of their life cycle (Poulin and Morand 2004). They also influence food web properties by modifying host behaviour, community structure, and population dynamics (Huxham et al. 1995; Thompson et al. 2005).

Host-parasite sub-systems are asymmetric webs (parasites feed on hosts). In these bipartite interaction networks, connectance can be simply considered as the number of realized links between parasites and their hosts divided by the number of potential links between parasites and hosts (Lafferty et al. 2006). Because the structure and internal properties of asymmetric webs are very different from those of symmetrical food webs, there is no reason to expect connectance to vary with species richness in host-parasite networks in the same way as it does in food webs. Some local host-parasitoids webs were intensively studied (e.g. Lewis et al. 2002) and Tylianakis et al. (2007) demonstrated that habitat modification may alter the structure of tropical host-parasitoid food webs. However, the lack of a large database of comparable webs has prevented the search of general patterns across scales with large variations in web size (number of host and parasite species). For instance whether or not the constant connectance hypothesis or any consistent pattern occurs in parasite-host webs has never been investigated. Here, we quantify empirical host-parasite web patterns on a large database of flea parasites infesting small mammals from 33 regions around the world forming a gradient of species richness. More specifically, we examine whether the level of connectance in mammal-flea webs varies with the size of the host-richness-by-parasiterichness matrix. This comparative analysis of host-parasite networks may reveal invariant patterns with deep implications for the stability and coevolutionary dynamics of these assemblages.

\section{Materials \& Methods}

Data were obtained from published surveys that reported flea distribution and abundance on small mammals (Didelphimorphia, Soricomorpha, Lagomorpha, and Rodentia) in 33 regions where we have at least 10 parasite species in order to achieve a large web in each locality (Appendix). Most published surveys on fleas parasitic on a small mammal community that were suitable for inclusion in our analysis come from the Palearctic, mainly from the area covered by the former Soviet

Union. This is because intense monitoring of fleas on small mammals to prevent possible outbreaks of plague was a common practice throughout this territory, but not elsewhere in the 
world. These sources provided data on the number of individuals of a particular flea species found on a number of sampled individuals of a particular host species. Single findings of a flea species on a host species or in a region were considered accidental and were not included in the analysis.

According to the constant connectance hypothesis, the relationship between the number of realised links (observed host-parasite connections $L$ ) and the number of potential links between parasites and their hosts $(P \times H)$ should be linear. According to some authors working on food webs (Brose et al. 2004, Beckerman et al. 2006) the relationship between realized links and species richness has an exponent between 1 (link-species scaling law) and 2 (constant connectance). Thus, the question is no longer whether the level of connectance is scale-invariant in networks of feeding interactions, but how steep is the decrease in connectance with species richness and what is the form of this relationship (Montoya and Sole 2003). Hence, we tested the linear model against several non-linear models (log-linear, exponential, or curvilinear) for their ability to describe better the relationship between $L$ and $\mathrm{PxH}$. The linear model is rejected only if the second order term of the quadratic regression is statistically significant. We also estimated the slope of the relationship between the number of realized links and species richness to follow the framework of Schmid-Araya et al. (2002).

Using host-parasite webs from the 33 different regions we estimated 3 different properties for each region (Figure 1):

The web connectance, which is the ratio between the number of realized links between fleas and their hosts divided by the number of potential links $(P x H)$ (Lafferty et al. 2006).

The mean diet overlap between flea species was calculated using pairwise Bray-Curtis similarity indices (Bray and Curtis 1957), which measure the mean overlap in host species used by any 2 parasites in the system.

The diet breadth of fleas; that is, their ability to infest (1) a large number and (2) a wide taxonomic range of host species (Poulin and Mouillot 2003), the latter providing an evolutionary perspective on host specificity. The taxonomic diversity of the host species used by a flea was measured using the index $S_{\mathrm{TD}}$, which represents the mean number of steps up a taxonomic tree that must be taken to reach a taxon common to 2 host species, computed across all possible pairs of host species used by a parasite (Poulin and Mouillot 2003). All mammal species infested by fleas were placed into a taxonomic structure (Wilson and Reeder 1993) with 5 hierarchical levels above species (i.e., genus, subfamily, family, order, and class).

We then searched for relationships between these variables and species richness across the 33 host-parasite interaction webs using linear and log-linear models. The significant relation with the highest R-square was retained.

\section{Results}

Our first result is the log-linear relationship between the number of fleas and the number of hosts (Figure 2A) assuming that parasite richness increases at a lower rate than host richness. We applied a quadratic regression to the relationship between the number of realised links (observed host-parasite connections, $L$ ) and the number of potential links between parasites and their hosts $(P \times H)$. Both the first order and the second order terms were statistically significant $(p<0.0001$ and $p=0.038)$, so we can reject the linear model. The best model (highest $R^{2}$ ) to describe the relationship between the number of realised links (observed host-parasite connections $L$ ) and the number of potential links between parasites and their hosts $(\mathrm{PxH})$ is the log-linear model (Figure 2B) demonstrating that the number of realised links increases at a slower rate that the number of the potential links, refuting the constant connectance hypothesis. More precisely, the slope of the logarithmic relationship between the number of realized links and species richness is 1.3, thus falling between 1 and 2 (Figure 2C).

We obtained an unambiguous decrease in the connectance of host-parasite webs along the host richness gradient across the 33 regions (Figure $3 \mathrm{~A}$ ). We got the same pattern using 
parasite richness as a predictor variable rather than host richness, as host and parasite richness are closely related $(r=0.56, p<0.001)$. We tested the species packing theory by seeking a relationship between host number in the region and the mean diet overlap among flea species (Figure 3B). As a result we found a significant log-linear decreasing relationship showing that, as species richness increases (either richness of parasites or hosts), fleas tend to reduce overlaps in resource use. This result may be due to some limits in diet breadth extension when host richness increases. Effectively the diet breadth of fleas in terms of number of host species infested tends to increase logarithmically with host richness (Figure 3C) while the diet breadth in terms of taxonomic diversity of the host spectrum is constant along the host richness gradient (Figure 3D).

\section{Discussion}

The influence of parasites on food web properties has been neglected for a long time despite the importance of parasites in food webs and their ability to modify the properties of their host assemblage (Huxham et al. 1995, Combes 1996, Thompson et al. 2005). Recently, Lafferty et al. (2006) pointed out an increasing connectance in food webs when parasite-host links are included. This result highlights that food webs are incomplete without parasites. Here we focused on parasite-host sub-systems to determine whether the connectance was constant along a gradient of species richness. Using a flea-mammal database from 33 regions of the world we demonstrated that the connectance of bipartite webs is not a constant along a gradient of species richness. However, we found another invariant-scaling relationship between parasite dietary breadth, in terms of host taxonomic diversity, and the number of host species (Figure 3D). Granted, our results are derived from published data on presence/absence, and these are not always a perfect reflection of existing relationships between hosts and parasites. However, we were able to exclude host-parasite associations based on poorly sampled individuals, which likely to be accidental rather than true biological interactions. Also, to generate the patterns that we found, accidental associations would have to be consistently biased in the same direction across most if not all our interaction webs, something that seems highly unlikely. Thus, our results are unlikely to be artefactual, and most probably reflect true ecological patterns.

We found decreasing connectance along the species richness gradient (Figure $3 \mathrm{~A}$ ); that is, additional potential connections between fleas and hosts are not actually realized when host richness increases. Even though fleas tend to reduce overlap in resource use when species richness increases (Figure 3B), we were unable to find any support for the species packing theory. Indeed, flea species are able to infest more host species in host-rich regional assemblages (Figure 3C) and do not reduce their diet breadth (Figure 3D) as predicted theoretically (MacArthur and Levins 1967). Thus the reduction of resource overlap observed in species-rich assemblages is simply due to the broader spectrum of hosts available for fleas rather than a species packing process. Accordingly, competition has never been identified as a significant structuring factor of these flea assemblages (Krasnov et al. 2005).

As an alternative, we suggest that some phylogenetic constraints on flea diet may explain our observed patterns. Indeed Cousins (1985) proposed that each consumer's diet is directly related to its phylogenetic origin because some morphological traits related to diet are conserved through lineages. Going a step further, Cattin et al. (2004) demonstrated, using 5 empirical food webs, that taxonomic affinity and the trophic similarities among species are strongly related within species communities, i.e. that closely-related species have some common ecological attributes allowing them to feed on the same kind of resources. In the case of host-parasite webs, the extent of the parasite diet is determined by the host specificity. A previous investigation on fleas suggests that host specificity is to a large extent phylogenetically constrained (Mouillot et al. 2006). As a consequence phylogenetic constraints on host specificity may impose limits on fleas preventing them from infesting more diverse host species in species-rich assemblages. The present observation stating that parasite diet breadth in terms of host taxonomic diversity is scale-invariant (see Figure 3D) is in perfect concordance with this explanation. Thus host-parasite connectance decreases along a richness gradient because flea species may be unable to increase the taxonomic 
diversity of the host species they exploit in host-rich assemblages. In support of this explanation, it has been found that in flea species that occur in different geographical regions, host specificity shows some plasticity, but this spatial variability in host specificity is unaffected by the size of the local pool of host species (Krasnov et al. 2004).

Our result is in accordance with results obtained from other asymmetric networks, specifically plant-animal mutualistic interaction webs. Jordano et al. (2003) suggest that constraints such as morphological mismatching or phenological uncoupling between mutualistic partners restrict the number of interactions established and thus prevent scale-invariance patterns to emerge by limiting the number of links. In parasite-host webs other variables can also influence the density of links in the network. Indeed the density of hosts may influence the transmission of parasites and can potentially influence the number of links within a parasitehost web but it was impossible to evaluate this variable at our scale of observation.

Surprisingly our result is in accordance with the most recent results obtained on food webs. Indeed a consensus is emerging suggesting that the relationship between the density of links and the number of species has neither an exponent of 2 (constant connectance) nor 1 (linkspecies scaling) (Montoya and Sole 2003, Brose et al. 2004, Beckerman et al. 2006). However, complete food webs and parasite-host webs are difficult to compare. In our case, the slope of 1.3, lower than those observed in food webs, suggests that the number of realized links increases less with species richness (Figure 2C). We suggest that connectance in host-parasite webs might be related to the necessary time-lag between an increase in host species richness and an increase in the number of parasites and thus the number of hostparasite associations (i.e., links) due to coevolutionary requirements. For instance we found that the number of flea species is not proportional to the number of host species, with fewer flea species than predicted for high levels of host richness (Figure 2A). Moreover we know that coevolutionary adjustments are likely to be much less important in standard food webs involving predators and prey (Thompson 1994). In particular, the establishment of a new hostparasite association requires time because a parasite has to adapt to the properties of each new host, such as resources provided by the host (e.g., blood composition), host anti-parasite defence strategies, those morphological (e.g., structure of pelage) and life history parameters (e.g., structure and microclimate of burrows) of a host that determine host availability for a parasite in space and time and the chances of completing the parasite's life cycle (e.g., survival of pre-imago), etc. Thus, connectance may not be able to respond to increasing species richness as rapidly in host-parasite systems as in classical predator-prey food webs. This prediction deserves to be tested using datasets with both prey-predator and parasitehost webs.

\section{Acknowledgements}

This study was partly supported by the Israel Science Foundation (Grant no. 249/04 to B. Krasnov \& G. Shenbrot). This is publication no. 236 of the Ramon Science Center and no. 579 of the Mitrani Department of Desert Ecology. We thank three anonymous reviewers and D.A. Kelt the Subject Matter Editor for their comments and constructive criticisms on our manuscript. 


\section{References}

Beckerman, A. P. et al. 2006. Foraging biology predicts food web complexity. - Proc. Nat. Acad. Sci. USA 103: 13745-13749.

Bray, J. R. and Curtis, J. T. 1957. An ordination of the upland forest communities of southern Wisconsin. - Ecol. Monogr. 27: 325-349.

Brose, U. et al. 2004. Unified spatial scaling of species and their trophic interactions. - Nature 428: 167-171.

Cattin, M. F. et al. 2004. Phylogenetic constraints and adaptation explain food-web structure. - Nature 427: 835-839.

Cohen, J. E. and Briand, F. 1984. Trophic Links of Community Food Webs. - Proc. Nat. Acad. Sci. USA 81: 4105-4109.

Combes, C. 1996. Parasites, biodiversity and ecosystem stability. - Biodiversity Conserv. 5: 953-962.

Cousins, S. H. 1985. The trophic continuum in marine ecosystems: structure and equations for a predictive model. - Can. J. Fish. Aquat. Sci. 213: 76-93.

Huxham, M., et al. 1995. Parasites and food web patterns. - J. Anim. Ecol. 64: 168-176.

Jordano, P. 1987. Patterns of mutualistic interactions in pollination and seed dispersal: connectance, dependence asymmetries, and coevolution. - Am. Nat. 129: 657-677.

Jordano, P. et al. 2003. Invariant properties in coevolutionary networks of plant-animal interactions. - Ecol. Lett. 6: 69-81.

Krasnov, B. R. et al. 2004. Geographical variation in host specificity of fleas (Siphonaptera) parasitic on small mammals: the influence of phylogeny and local environmental conditions. Ecography 27: 787-797.

Krasnov, B. R. et al. 2005. Abundance patterns and coexistence processes in communities of fleas parasitic on small mammals. - Ecography 28: 453-464.

Lafferty, K. D. et al. Parasites dominate food web links. - Proc. Nat. Acad. Sci. USA 103: 11211-11216.

Lewis, O. T. et al. 2002. Structure of a diverse tropical forest insect-parasitoid community. - J. Anim. Ecol. 71: 855-873.

MacArthur, R. and Levins, R. 1967. Limiting Similarity Convergence and Divergence of Coexisting Species. - Am. Nat. 101: 377-387.

Marcogliese, D. J. and Cone, D. K. 1997. Food webs: a plea for parasites. - Trends Ecol. Evol.12: 320-325.

Martinez, N.D. 1991. Artifacts or Attributes - Effects of Resolution on the Little-Rock Lake Food Web. - Ecol. Monogr. 61:367-392.

Memmott, J. et al. 2000. Predators, parasitoids and pathogens: species richness, trophic generality and body sizes in a natural food web. - J. Anim. Ecol. 69: 1-15.

Montoya, J. M. and Sole, R. V. 2003. Topological properties of food webs: from real data to community assembly models. - Oikos 102: 614-622. 
Mouillot, D. et al. 2006. Conservatism of host specificity in parasites. - Ecography 29: 596602.

Poulin, R. and Mouillot, D. 2003. Parasite specialization from a phylogenetic perspective: a new index of host specificity. - Parasitology 126: 473-480.

Poulin, R. and Morand, S. 2004. Parasite Biodiversity. Smithsonian Books, Washington, DC, USA.

Schmid-Araya, J. M. et al. 2002. Connectance in stream food webs. - J. Anim. Ecol. 71: 10561062.

Thompson, J.D. 1994. The Coevolutionary Process. University of Chicago Press, Chicago.

Thompson, R. M. et al. 2005. Importance of parasites and their life cycle characteristics in determining the structure of a large marine food web. - J. Anim. Ecol. 74: 77-85.

Tylianakis, J. M. et al. 2007. Habitat modification alters the structure of tropical host-parasitoid food webs. - Nature 445: 202-205.

Warren, P. H. 1989. Spatial and Temporal Variation in the Structure of a Fresh-Water Food Web. - Oikos 55: 299-311.

Wilson, D. E. and Reeder, D. M. 1993. Mammal species of the world: A taxonomic and geographic reference. Smithsonian Institution Press, Washington.

Winemiller, K. O. 1989. Must Connectance Decrease with Species Richness. - Am. Nat. 134: 960-968. 

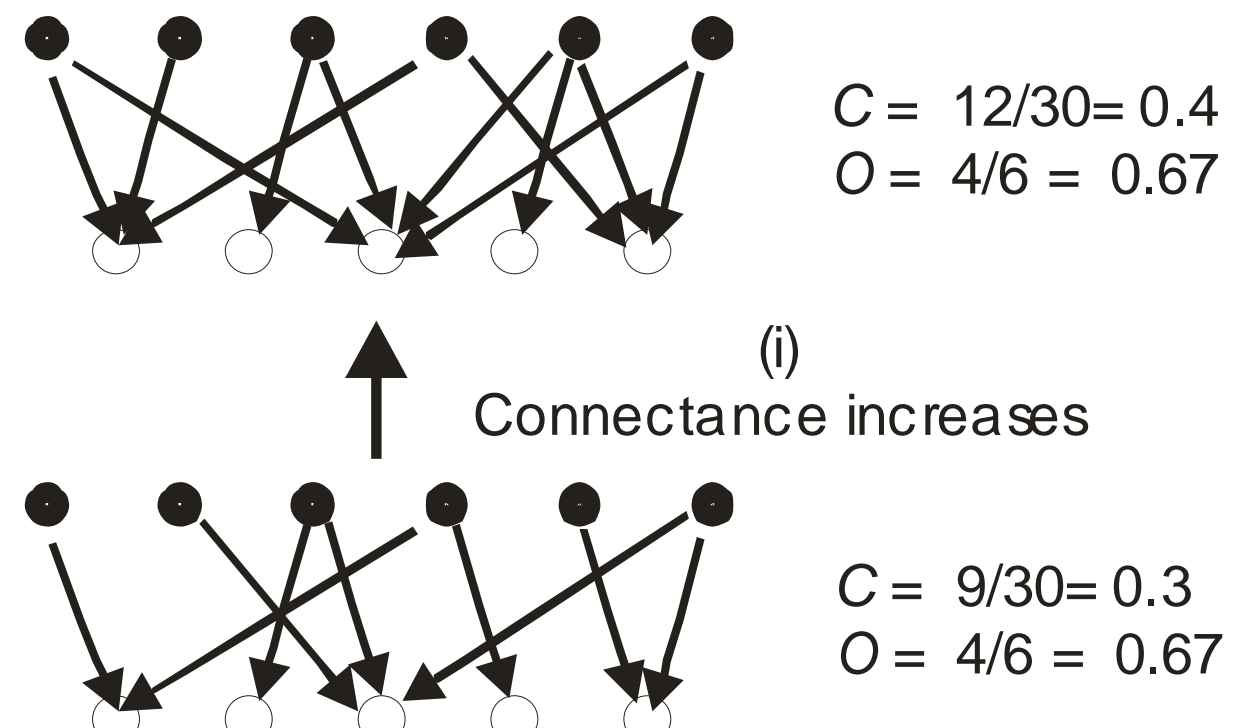

(ii)

\section{Overlap increases}

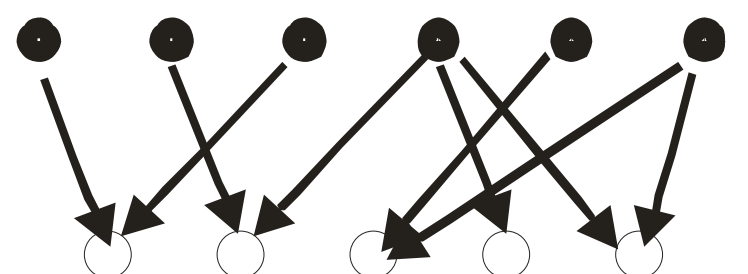

$C=9 / 30=0.3$
$O=5 / 6=0.83$

Figure 1. A hypothetical example in which one property is changed from the central web to produce the 2 satellite webs. Black circles are parasites while blank circles are hosts. These 2 independent properties of host-parasite webs are (i) the connectance $(C)$ which is assimilated to the number of realized links between fleas and their hosts divided by the number of potential links between parasites and hosts $(P \times H)$, and (ii) the mean diet overlap $(O)$ between parasites which is basically estimated by the proportion of parasites having only hosts in common with other parasites. 

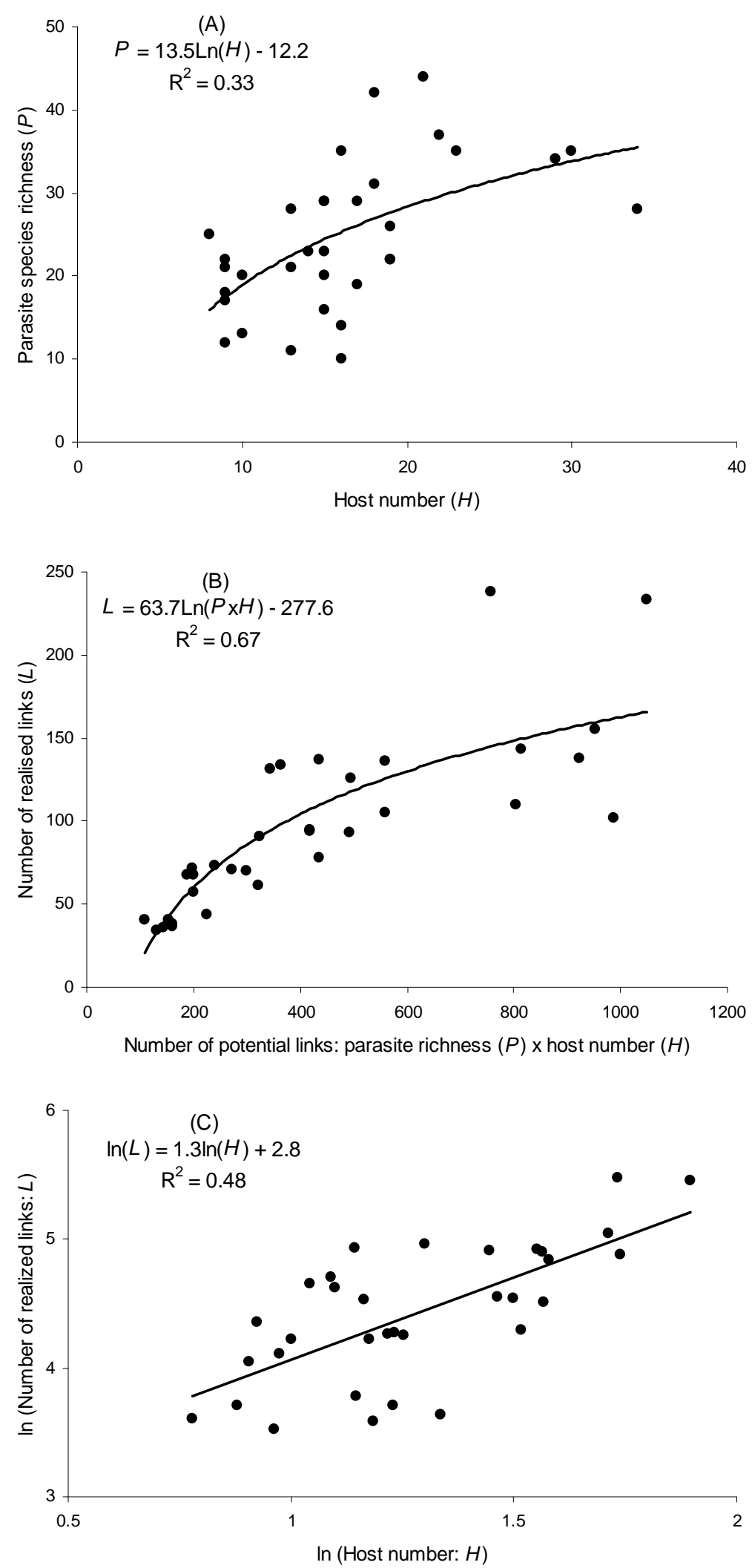

Figure 2. Observed relationships between host number and flea richness (A), between the number of potential links and the number of realized links in host-parasite webs (B) and between the number of host species and the number of realized links (C, In-In regression) across 33 regions. 

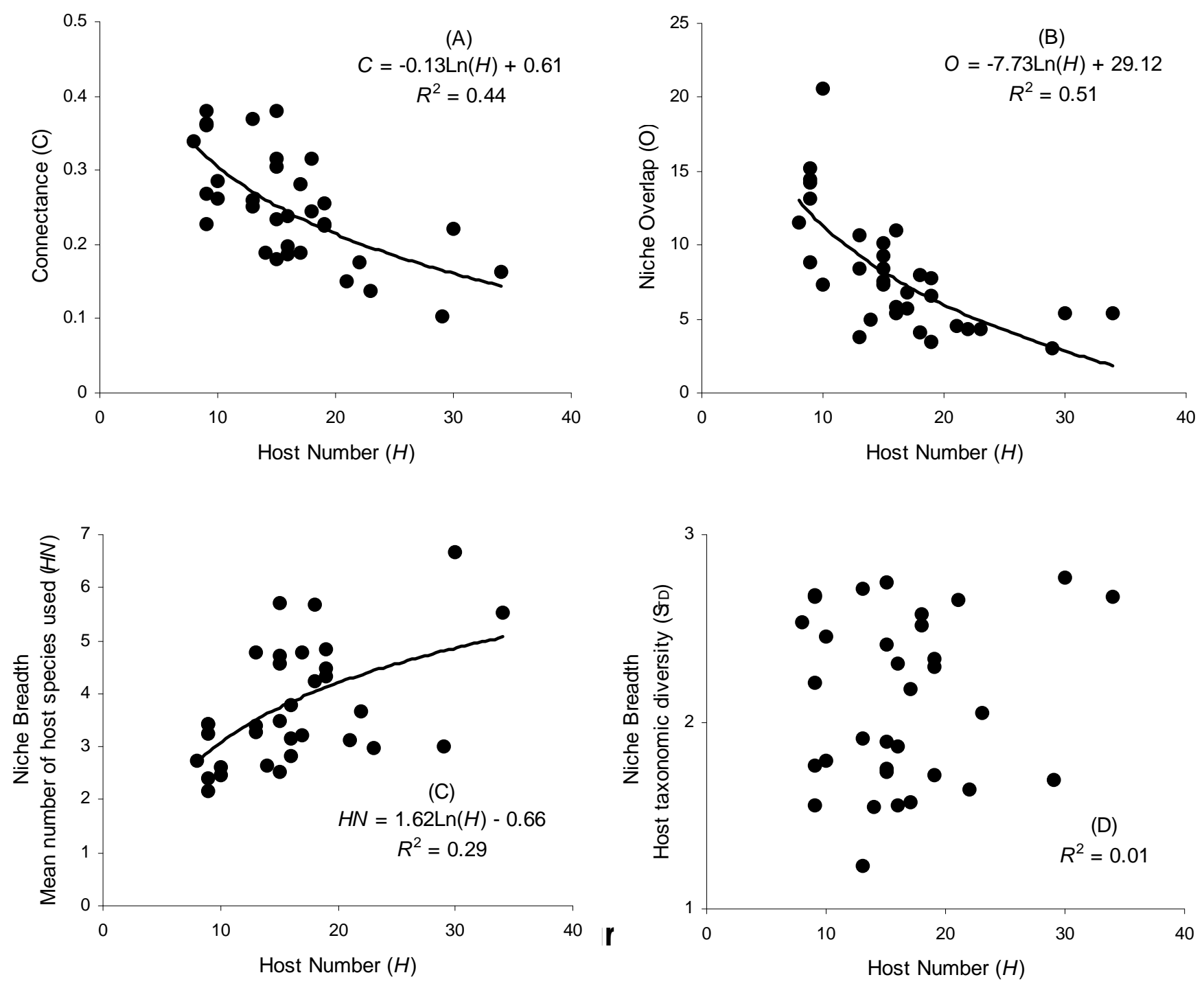

Figure 3. Relationships between host number and connectance of host-parasite webs, mean diet overlap among fleas and mean diet breadth of fleas across 33 regions. Trend lines are indicated where a linear or a log-linear model fits significantly. 